\title{
Regionalprobleme in der Schweiz Einleitung in das Themenheft
}

Der 36. Europäische Kongreß der Regional Science Association findet vom 26. bis 30. August in Zürich statt. Diese internationale Tagung bildet den äußeren Anlaß, das Heft 2/96 der "Geographica Helvetica» als regionalwissenschaftliches Themenheft zu konzipieren. Ziel dieses Heftes ist es, der Leserschaft dieser einzigen gesamtschweizerischen geographischen Fachzeitschrift in Form von Kurzaufsätzen einen knappen Überblick über aktuelle Regionalprobleme in der Schweiz zu vermitteln. Es war die Absicht der Herausgeber, im beschränkt zur Verfügung stehenden Platz möglichst viele Autoren zu Wort kommen zu lassen, wobei wir uns bewußt sind, daß wir leider auch so nur einen Teil der schweizerischen Regionalwissenschafter berücksichtigen konnten.

Auch wenn dies den tagespolitischen Aktualitäten und den Meldungen in den Massenmedien über die Globalisierung und Internationalisierung in Wirtschaft, Gesellschaft und Kultur teilweise widerspricht, sind wir der Überzeugung, daß die Beschäftigung mit regionalwissenschaftlichen Fragestellungen keineswegs anachronistisch geworden ist. Beispielsweise sind wirtschaftliche Disparitäten zwischen verschiedenen Teilräumen eines Landes weiterhin ein aktuelles Thema; insbesondere in der föderalistischen Schweiz, wobei sich hier die Optik etwas vom Klein- zum Größerräumigen verschoben hat. Die politischen Auseinandersetzungen um die Linienführung der Neuen Eisenbahn-Alpentransversalen (Neat) oder die Herabstufung des Flughafens Genf Cointrin gegenüber Zürich Kloten durch die Swissair zeugen davon.

Es ist unverkennbar, daß sich parallel zur Globalisierung auch Prozesse der Regionalisierung abspielen. Auf der subnationalen Ebene melden sich unter Berufung auf geschichtliche Wurzeln regionalistische Bewegungen vermehrt zu Wort. Die Neubelebung der Regionen hat sicher auch damit zu tun, daß gerade im Prozeß der Globalisierung verstärkt ein Bedürfnis nach Heimat, Überschaubarkeit und Zugehörigkeit entsteht. Im wirtschaftlichen Bereich wird der Region als «innovativem Milieu» oder «Netzwerk» zunehmend Beachtung geschenkt, d. h., daß eben nicht alle örtlichen Verankerungen der Wirtschaft verschwinden, sondern daß viele wirtschaftliche Tätigkeiten an "Cluster» aus verwandten Betrieben, Ausbildungsstätten und anderen Infrastrukturen gebunden sind. Durch die schwindende Bedeutung nationaler Grenzen sind von diesen Regionalisierungsprozessen auch häufig Grenzregionen erfaßt. Auf der übernationalen Ebene stellen wir eine andere Art von Regionalisie- rung fest, indem sich neue wirtschaftliche Großregionen herausbilden (Nafta, Asean, EU, Mercosur).

Der Erscheinungszeitpunkt dieses Themenheftes liegt günstig. Wir stehen in bezug auf die Regionalprobleme in der Schweiz und die schweizerische Regionalpolitik in einer ausgesprochenen Phase des Umbruchs. Obwohl oder vielleicht gerade weil sich die Schweiz auf der politischen Ebene starrköpfig gegen die Internationalisierung stemmt, ist sie im wirtschaftlichen Bereich davon mit aller Wucht getroffen. Der bisher geschützte Agrarsektor muß in kurzer Zeit aufgrund der Gatt/WTO-Verpflichtungen liberalisiert werden. Die Industrie befindet sich in einem unerbittlichen weltweiten Wettbewerb und reagiert mit einschneidenden Restrukturierungen. Diese Prozesse werden verstärkt durch die starke Aufwertung des Schweizerfrankens, wodurch wertschöpfungsschwache Branchen - u. a. auch gewisse Sparten des Tourismus - in arge Nöte geraten. All diese Strukturwandlungen haben regionalwirtschaftliche Implikationen. Die großstädtischen Zentren, insbesondere der Großraum Zürich, scheinen dadurch bevorteilt, während agrarisch, industriell oder touristisch geprägte Räume abseits der Großagglomerationen mit eher ungünstigen Entwicklungsaussichten konfrontiert sind. Dies sind Entwicklungen, die in einem föderalistisch geprägten Land wie der Schweiz staatspolitisch nur schwer verträglich sind und zu beträchtlichen Spannungen im Innern führen.

Der Staat ist seit einigen Jahren daran, seine Raumordnungspolitik den neuen Verhältnissen anzupassen. Die neue Raumordnungspolitik nimmt nun greifbare Konturen an. Aller Voraussicht nach werden dieses Jahr auf Bundesebene die Neuorientierung der Regionalpolitik (im Verantwortungsbereich des Bundesamtes für Industrie, Gewerbe und Arbeit) und die Grundzüge der Raumordnung Schweiz (im Verantwortungsbereich des Bundesamtes für Raumplanung) verabschiedet. Besonders ist hervorzuheben, daß nicht allein die regionale Wirtschaftspolitik reorganisiert wird, sondern daß ein vermehrtes Zusammengehen mit der Raumplanung angestrebt wird, mit dem Ziel, eine zukunftstaugliche Raumordnung zu gestalten, die auch den am Rio-

Daniel Wachter, PD Dr., Bundesamt für Raumplanung, Einsteinstraße 2, 3003 Bern; Hans Elsasser, Prof. Dr., Geographisches Institut, Universität Zürich Irchel, Winterthurerstraße 190, 8057 Zürich 
Umweltgipfel von 1992 aufgestellten Kriterien der Nachhaltigkeit genügt.

Die einzelnen Artikel in diesem Themenheft wurden aufgrund folgender Überlegungen angeordnet. Zu Beginn plazierten wir drei Beiträge mit Grundsatzcharakter. Der Beitrag von Stephan Bieri gibt einen umfassenden Überblick über die neue Regionalpolitik der Schweiz. Diese Informationen bieten eine ausgezeichnete Grundlage für die weiteren Artikel zu einzelnen Spezialthemen. Ebenfalls gesamtschweizerisch und staatspolitisch ausgerichtet ist der Beitrag von Martin Lendi über Föderalismus und Regionalpolitik. Martin Boeschs Darlegungen über Städte und Agglomerationen haben u. E. Grundsatzcharakter, weil sie über die Agglomerationsproblematik hinaus das Selbstverständnis der Schweiz thematisieren, verbunden mit der Forderung, daß die Schweizer endlich auch mental den funktional längst erfolgten Übergang von der Bauernschweiz zur Stadt Schweiz nachvollziehen sollten.

Danach folgt ein Dreierblock, der sich schwergewichtig mit regionalwirtschaftlichen Fragen der Industrie beschäftigt. Christian Hanser und Jürg Kuster vertreten die wohl zu Recht als staatspolitisch schicksalshaft bezeichnete These, daß der sich aufwertende «Zürcher Finanzfranken» die industriell-gewerbliche, die touristische und landwirtschaftliche Wirtschaftsbasis der übrigen Schweiz erodiere. In offenkundigem Gegensatz dazu steht der optimistische Beitrag von Denis Maillat, der in der ehemals monostrukturierten Uhrenregion im Jura markante industrielle Revitalisierungsphänomene in die Richtung von innovations- und wertschöpfungsstarken Aktivitäten erkennt. Der Artikel von Beat Hotz-Hart ergänzt denjenigen von Denis Maillat bestens, indem er in etwas allgemeinerer Art die technologiepolitischen Grundlagen eines möglichen industriellen Wiederaufschwungs - auch abseits der großen städtischen Zentren - schildert.
Es folgen drei Beiträge zu Problemen des ländlichen und Landschaftsraumes. Paul Messerli beschäftigt sich mit den Auswirkungen der umgebauten Regionalpolitik auf die Berggebiete. Peter Rieder legt dar, wie sich die reorganisierte Landwirtschaftspolitik auf die verschiedenen Regionen des Landes und auf die natürliche Umwelt auswirkt. Willy A. Schmid widmet sich der ökologischen Planung. Es scheint uns wichtig, daß in diesem regionalwissenschaftlichen Themenheft auch ökologische Fragen aufgegriffen werden - in Übereinstimmung mit dem auf der politischen Ebene eingeschlagenen Weg in Richtung einer "nachhaltigen Raumordnung».

Einen weiteren thematischen Bereich bilden die beiden Artikel von Remigio Ratti und Maria Lezzi zum Phänomen der sich neu strukturierenden Grenzregionen. Ans Ende der Beiträge haben wir denjenigen von Martin Schuler gesetzt; keinesfalls aus Geringschätzung, sondern weil er u.E. in gewisser Weise eine Synthese der übrigen Beiträge bildet, ist die regionale Bevölkerungsentwicklung doch in ausgeprägtem Maße die Folge der in den vorangehenden Artikeln beschriebenen Prozesse.

Wir hoffen, daß es uns mit diesem Themenheft über die Einzelinformationen hinaus gelungen ist, zumindest teilweise die Vielfalt und Breite der Regionalwissenschaften deutlich zu machen. Die Begriffe "Vielfalt» und «Breite» beziehen sich auf unterschiedliche Sachverhalte: Einmal geht es um die Disziplinen, die in den Regionalwissenschaften vertreten sind (Ökonomie, Rechtswissenschaften, Ingenieurwissenschaften, Geographie usw.). Zum zweiten geht es um die unterschiedlichen Aktivitäts- und Berufsfelder wie Hochschulen, öffentliche Verwaltung, parastaatliche Organisationen und Privatwirtschaft. Schließlich freut es uns, daß sich in diesem Heft auch die sprachliche Vielfalt der Schweiz niedergeschlagen hat. Wir hoffen, daß wir so dem transdisziplinären Charakter der Regionalwissenschaften gerecht werden und daß wir mit diesem Themenheft auch einen kleinen Beitrag zur Förderung der Netzwerkstruktur der Regionalwissenschaften in der Schweiz leisten können. 